\title{
Un problème d'ordre des mots en chinois vernaculaire : quand et où s'est produit le changement shuo ta bu guo > shuo bu guo ta.
} Christine Lamarre

\begin{abstract}
This paper aims to show when and where oecured the syntactic change which led from the word order "Verb + Object + negation + Resultative postverb" (here "V O Nég v", ex : shuō tā bú guò, prevailing in Tang times, to the present Pekingese word order "Verb + negation + Resultative postverb + Object" (here "V Neg v O, ex : shuō bú guò tā). While both orders are met in 'baihua' literature of Ming times, we argue - through an investigation of Tang, Song, and Yuan texts - that the present Pekingese word order was the only attested one in the Northern dialect as soon as the Yuan period ; the ancient word order " $\mathrm{0}$ Neg v", however, is still very common in many Southern dialects, which accounts for the coexistence of "V 0 Neg v" and "V Neg $\checkmark 0 "$ in Ming texts. This word order change is linked to the one that occuring later in corresponding affirmative constructions. The significance of both changes lies in the fact that Modern Pekingese is thus provided with two distinct structures unambiguous as for resultative and potential meaning, while the previous constructions were basically resultative acquiring only in some limited contexts a potential meaning.
\end{abstract}

\section{Citer ce document / Cite this document :}

Lamarre Christine. Un problème d'ordre des mots en chinois vernaculaire : quand et où s'est produit le changement shuo ta bu guo > shuo bu guo ta.. In: Cahiers de linguistique - Asie orientale, vol. 14 1, 1985. pp. 83-98;

doi : 10.3406/clao.1985.1168

http://www.persee.fr/doc/clao_0153-3320_1985_num_14_1_1168

Document généré le 02/06/2016 


\section{UN PROBLEME D'ORDRE}

\section{DES MOTS EN CHINOIS}

\section{VERNACULAIRE : QUAND ET OU}

\section{S'EST PRODUIT LE CHANGEMENT}

\section{SHUO TA BUGUO > SHUO BU GUO TA}

\section{INTRODUCTION}

Nous souhaitons tirer au clair ici un point précis d'histoire du

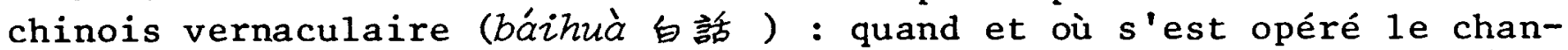
gement d'ordre des constituants dans les constructions du type shuöbuguò tā 説不遇他 "ne pas avoir le dernier mot sur 1ui, ne pas arriver à 1 'emporter sur lui dans une discussion" ou guàibude tấ 怪不得 他 "ne pas pouvoir lui en vouloir" ?

En effet, en pütōnghuà (langue standard, fondée sur le pékinois), la norme est shuōbuguó t $\bar{\alpha} ; 1$ 'objet $t \bar{a}$ est situé après la construction potentielle shuóbuguó (à 1 'affirmative shuōdeguò). Mais dans la langue vernaculaire ancienne (zăoqi báihuà 早期白話) telle que la reflètent des romans comme le Shuǐ Hŭ Zhuàn ou le Jín Píng Méi, 1'ordre inverse shuō tā bú guò 説 他 不通 - où 1 'objet tā est inséré entre le verbe principal et la négation bù - était aussi couramment utilisé. Ce dernier ordre est en outre attesté aussi dans plusieurs dialectes méridionaux contemporains. Il est donc indispensable, pour éclaircir ce point d'histoire (quand s'est produite 1 'unification en shuobuguo $t a$, ordre actuel du pékinois), de ne pas oublier la dimension géograhique, dialectale, du problème.

Si nous avons privilégié ce changement d'ordre précis, aux dépens d'autres de même ampleur (par exemple le déplacement de la négation dans les structures "bù shuō de 不説得 + objet" > "shuobude + objet"), c'est qu'il représente pour nous un exemple de la vaste réorganisation du chinois visant à donner à la distinction sémantique entre potentiel et accomp1i - repérable auparavant grâce au contexte - une expression syntaxique dénuée d'ambiguité.

Les constructions que nous prenons ici en compte comprennent : un verbe principal $\mathrm{V}$; son objet $\mathrm{O}$; une négation Nég ; un verbe complémentaire marquant le résultat du verbe principal, pour former avec lui une construction résultative, et que nous noterons $v$. Cette C.L.A.O. Vo1. XIV N ${ }^{\circ} 1$ Juin 1985, pp. 83-98. 
catégorie des $\mathrm{v}$ comprend aussi le verbe de 得, mais celui-ci présente certaines caractéristiques qui le distinguent des autres $v$ dans les premiers stades de son évolution (par exemple, la possibilité, à une époque il est vrai limitée, pour "v" et "Nég + $D E$ " d'être en co-occurence dans de telles constructions). Nous préférons, pour le moment, symboliser de 得 par $D E$, étant bien entendu qu'à partir des Yuan $1 \mathrm{a}$ graphie 的 se recontre aussi.

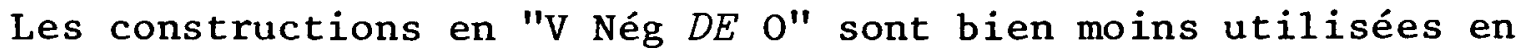
pékinois actuel que celles en "V Nég v O", le de 得 potentiel post-verbal ayant beaucoup perdu de sa vigueur au profit des auxiliaires préverbaux néng能 ou kĕy゙̌ 可以. Ainsi, 1 'exemple cité plus haut guàibude $t \bar{a}$ 怪不得他 "ne pas pouvoir lui en vouloir" se dirait plutôt aujourd' hui bù néng guài tā 不能怪他, la forme guàibude étant réduite à son emploi idiomatique "pas étonnant si ...". En langue vernaculaire ancienne, cependant, de 得 post-verbal avait un sens très étendu, comme potentiel, même à l'affirmative. Cf. I'exemple suivant du Lăo Q (I, p. 92) :

（1）他漠兒言言吾説不得的

tà hàn'ér yányǔ shuó bu de de

il - chinois - parler - Nég - DE - part. mod.

"Il ne sait pas parler le chinois".

où $D E$ correspond au hui 會 du chinois contemporain.

Avant de passer à 1 'étude des occurrences de ces deux ordres dans divers documents de chinois vernaculaire ancien, examinons d'abord 1'origine de ces constructions et leur évolution jusqu'au Xe siècle de notre ère environ.

1. POURQUOI DEUX ORDRES POSSIBLES : "V O NEG $v "$ / "V NEG $v$ O" ? APERCU HISTORIQUE DE LA CONSTRUCTION.

1.1. Cette construction a pour origine la construction causative (appelée shǐchéngshi 使成式 par Wang Li) ou la construction résultative (jiéguó bǔyú 結果補語) qui existait déjà en langue classique (1):

（2）射殺之（史記：項羽本紀）

shè shā zhì

tirer sur - tuer - le

"1e tuer d'une flèche".

（3）射之死（左傅：昭公21）

shè zhi $s i z$

tirer sur - lui - mourir

"idem".

(1) Cf. Zhou Chiming (1958) 
Le premier exemple a pour ordre "V v O" et constitue ce que Zhou Chiming (1958) appelle la "forme groupée" (héyòngshi 合用式); c'est 1 'ordre du pékinois actuel. Le deuxième exemple a 1 'ordre "V O v" où O est en fait pivot (jiānnyน̌ 兼語, objet de $v$ et sujet de $v$ ) et constitue ce que Zhou appelle la "forme disjointe" (fényòngshi 分用式); les verbes formant la construction résultative sont séparés par 1 'objet. Ce dernier ordre n'est plus admis en pékinois. Le premier a pu longtemps s'interpréter comme une simple juxtaposition de deux propositions du type "V er V 0 " :

(4) 射而䄫之 shè ér shä zhi

tirer sur - et - tuer - lui

"tirer sur lui et le tuer".

Le deuxième ordre est une construction à pivot (jīânyǔshì.兼語式).

1.2. La possibilité d'insérer la négation bù 不 ou de 得 dans ce type de constructions intervient plus tard. C'est d'abord la forme négative qui se développe peu à peu, avec une longue période de transition entre la simple juxtaposition évoquée plus haut, le deuxième élément niant seulement le premier :

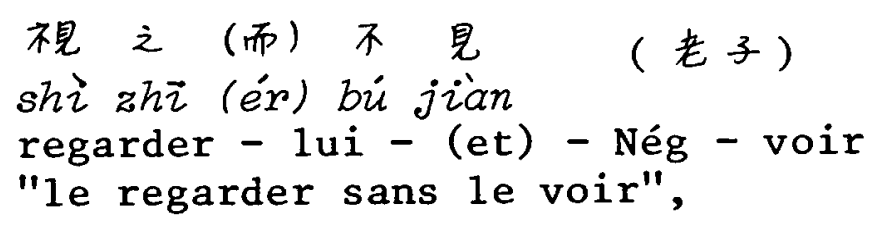

et une construction exprimant 1 'impossibilité :

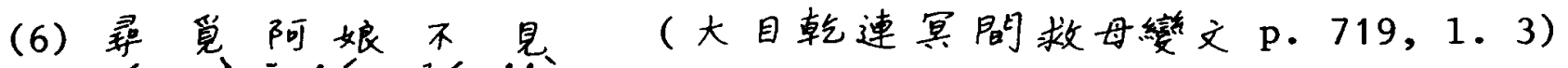 xún mi āniáng bú jiàn

qui correspond au pékinois "zhăobujiàn 找不見 + O". C'est sous les Tang que cette forme négative peut avoir un sens potentiel très net, mais l'ordre "V O Nég v" laisse toujours la porte ouverte à une interprétation comme simple négation si le contexte 1 'exige, d'autant plus qu'à 1 'époque on ne dispose pas encore de 1 'opposition bù 不 / mé $i$ 没. C'est aussi à ce moment-1à qu'apparâ̂t 1 'ordre du pékinois actuel "V Nég v O", mais il est encore très rare. Ota Tatsuo (1958, p. 234) note que sous les Tang et les Cinq Dynasties, 1 'usage de "V Nég v" pour exprimer 1 'impossibilité est déjà assez courant, mais que 1 'objet est rarement présent. Si 1 'objet apparâ̂t, les deux ordres "V 0 Nég $v$ " et "V Nég $v 0$ " sont attestés, mais le second est extrêmement rare. Zhou Chiming (1958) ne donne pas de date précise pour 1'apparition de la forme "V Nég $v \mathrm{O}^{\prime}$ et les exemples qu'il cite ne sont pas antérieurs aux Ming. I1 fait remonter " $V$ Nég $v$ " à 1 'époque des $S i x$ Dynasties (Ve - VIe siècles). 
1.3. Pour ce qui est de la forme affirmative de notre construction, "V $D E$ v O", en pékinois (exemples : shuōdeguo tā 説得過他"pouvoir 1 'emporter sur lui" ou chídexià fdn 吃得 $F$ 飯 "arriver à manger, avoir de 1 'appétit"), sa forme $1 \mathrm{a}$ plus répandue, à l'époque, était encore "V $D E O \mathrm{~V}$ " ("V1 $D E O \mathrm{~V} 2$ "), où o était pivot, objet de V (V1) et sujet de $v$ (V2) (2).

Cette construction affirmative présente une ambiguỉté structurelle qui a ensuite été levée par une évolution en deux constructions distinctes : la construction potentielle (kènéng bǔyǔ 可能補語 ou büyü de kénéngshi 補語的可能式) d'une part, la construction à complément de résultat ou de degré (jiéguó büyŭ 結果補語 ou chéngdù bǔyŭ 程度補語), d'autre part. A 1 'époque, 1 'interprétation potentielle ou résultative (ou descriptive) dépendait essentiellement du contexte et des relations sémantiques entre les constituants de cette construction.

1.4. La construction "V $O$ Nég $D E$ " est la seule attestée jusqu'aux Song. Ensuite, la forme "V Nég $D E$ O" apparâ̂t ; d'abord minoritaire, elle se répand progressivement, allant jusqu'à 1 'emporter en pékinois actuel. Öta Tatsuo (1958) constate à ce sujet : "Sous les Tang..., bùdé 不得 se plaçait nécessairement derrière le complément d'objet, quelle que fût la longueur de celui-ci. C'est sous les Song qu'apparut bùdé placé immédiatement derrière le verbe, sans en être séparé par le complément d'objet" (3). Dans ce cas aussi, nous pouvons constater que les formes les plus anciennes "V O Nég $D E$ " peuvent s'interpréter avec un sens plein pour "Nég $D E$ " : "ne pas obtenir". Nous avons donc encore un cas de juxtaposition. Gen Sachiko (1985) a relevé plusieurs constructions, avec ce sens, dans les biànwén. Certes, le sens potentiel est apparu très tôt (4), mais 1 a structure était ambiguë et laissait $1 a$ possibilité d'une interprétation "V O ér 而 Nég $D E$ O".

Pour résumer, nous avons donc sous les Tang 1 'ordre "V O Nég v" ou "V O Nég $D E$ " en position largement dominante, même si 1 'on trouve déjà quelques (rares) occurrences de "V Nég v O". A travers divers documents en langue vernaculaire, nous allons maintenant examiner $1 \mathrm{e}$ processus qui a conduit à renverser ce rapport de force et à 1'unification en "V Nég v O" ou "V Nég $D E$ O" en pékinois contemporain. Mais, auparavant, faisons d'abord une brève incursion dans les dialectes.

\section{2. "V O NEG $v ">$ "V NEG $v O "$ : LA VARIETE DIALECTALE}

La question du passage de 1 'ordre "V O Nég v" à "V Nég v O" se pose en diachronie, mais aussi en synchronie, puisque bon nombre de dialectes ont conservé cet ordre " $V$ O Nég $v$ ". Nous ne ferons pas ici

(2) Cf. Lü Shuxiang (1944); Ōta Tatsuo (1958, pp. 401 - 402).

(3) Cf. ōta Tatsuo (1958, p. 232).

(4) Cf. Ōta Tatsuo (1958, p. 231). 
le bilan exhaustif de la répartition géographique de ces deux variantes d'ordre des mots; nous nous contenterons de rappeler quelques remarques effectuées à ce propos dans trois ouvrages :

- M. Hashimoto (1978) attire notre attention sur les convergences entre les évolutions latitudinale (du nord au sud de la Chine) et longitudinale (de la langue classique au chinois contemporain), entre autres pour les constructions causatives. Il note à ce propos que les dialectes Wu et Hakka, par exemple, ont conservé 1 'ordre "V O Nég v". Ainsi, 1 'équivalent du pékinois shuōbuguò tā 説不過他"ne pas avoir le dernier mot sur lui" se dit : en Wu : 話伊物過 (dialecte de Shanghai), en Hakka : 講佢唔赢 (dialecte de Hailu 海陸) (5).

- Yuan Jiahua (1983, p. 321) note aussi la grande "souplesse" des dialectes en matière d'ordre des mots et cite le même exemple de dialecte Wu ainsi qu'un exemple de dialecte Gan 竡, qui présente un ordre extrêmement bizarre que nous $n^{\prime}$ avons trouvé dans aucun texte historique, mais que nous vu par ailleurs décrit pour le parler de Wuyi 武義 dans le Zhejiang 浙江 (cf. Fu Guotong, 1961), et que Zhan Bohui (1981, p. 78) affirme aussi exister à Suzhou蘇州, c'est-à-dire 1'ordre "V Nég O v". Yuan Jiahua estime également qu'en pékinois parlé, on rencontre aussi shuō tā bú gù 説他不通, mais aucun de nos informateurs n'a pu nous confirmer cette occurrence. Il faudrait certainement mener une enquête plus étendue et diversifiée. Il convient de distinguer les archaĩsmes et expressions figées de 1 'ordre "productif" dans une conversation spontanée. L'ordre productif, en pékinois, nous semble bien être "V Nég v O".

A 1 'inverse des archaismes, il ne faut pas sous-estimer 1 'influence du putonghua sur les jeunes des zones dialectales; un jeune informateur shanghaīen nnus a appris que sa génération, tout en acceptant bien sûr "V O Nég v", trouve certainement cette tournure vieillotte ou "paysanne" et utilise aussi beaucoup 1 'ordre pékinois "V Nég $v O^{\prime}$. Il semble aussi que le fait que 0 soit un pronom personnel améliore considérablement 1 'acceptation de "V 0 Nég $v$ " (ce facteur ne semble pas entrer en ligne de compte, sous les Song, par exemple).

(9):

Ce même informateur de Shanghai accepte ainsi (7) et (8), mais non

（7）誨等週

parler - lui - négation - passer

"ne pas 1'emporter (oralement) sur 1ui".

（8）打伊勿展

battre - lui - négation - mourir

"le battre sans arriver à le tuer".

（9）*打狛可死

battre - chien - négation - mourir

"battre le chien sans pouvoir le tuer".

(5) Cf. M. Hashimoto (1978, pp. 50 - 52). 
Là aussi, les faits demandent à être examinés plus en détail ; 1a phrase-type des enquêtes dialectales étant précisément shuobuguo $t a$, les cas où 0 n'est pas un pronom personnel sont souvent passés sous silence.

- Zhan Bohui (1981) cite aussi plusieurs exemples de dialectes. En cantonais :

$$
\begin{aligned}
& \text { (10) 我 打 } \frac{\text { 唔 }}{\text { je }} \text { - battre }- \text { négation - passer - lui } \\
& \text { "je ne peux pas l'emporter sur lui au combat". }
\end{aligned}
$$

$$
\begin{aligned}
& \text { (11) 我 打 佢 } \\
& \text { je - battre - 唔 } \\
& \text { "idem". }
\end{aligned}
$$

Les deux ordres sont donc attestés ; en pékinois, seul dăbuguio tã est possible.

En dialecte de Chaozhou:

$$
\begin{aligned}
& \text { (12) 我 呾 } 1 \text { 伊 唔 } \\
& \text { je - parler - lui - négation - passer } \\
& \text { "je ne peux pas l'emporter (oralement) sur lui", } \\
& \text { correspond au pékinois shuōbuguio tá. } \\
& \text { En dialecte de Suzhou: }
\end{aligned}
$$

$$
\begin{aligned}
& \text { （13）我講伊迥 } \\
& \text { je - parler - 1ui - négation - passer } \\
& \text { "je ne peux pas } 1 \text { 'emporter (oralement) sur lui" }
\end{aligned}
$$

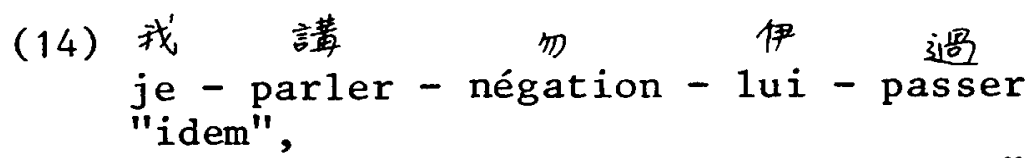

les deux ordres "V o Nég v" et "V Nég O v" sont possibles. Rappelons, à propos de ce dernier ordre que nous ne 1 'avons jamais rencontré dans d'autres descriptions du parler de Suzhou. Et Zhan Bohui ne cite pas ses sources $(6)$.

3. "V O NEG $v ">$ "V NEG $v$ O" : QUAND ET OU ? ANALYSE DES TEXTES VERNACULAIRES

3.1. Miyata Ichiro (1973, pp. 44 - 45) fait un bilan du problème concernant les deux ordres "V O Nég v" et "V Nég v O" dans les grands romans en báihuà. Nous résumons ci-dessous son analyse.

Miyata note d'abord qu'en pékinois, les constructions du type shuōbuguó tā 説不過他"ne pas avoir le dernier mot sur lui", bǐbushàng $t \bar{a}$ 比不上他"ne pas le valoir" ou duibuqz $z^{2} z^{2}$ "( $\mathrm{je} \mathrm{m}^{\prime}$ ) excuse envers toi", etc. suivent toutes 1 'ordre "V Nég $v 0$ " ; 1'ordre "V O Nég v" a une forte coloration dialectale. Il fait ensuite remarquer que les

(6) Pour les exemples cités par Zhăn Bóhuì, cf. Zhăn Bóhuì (1981, pp. $77-78)$. 
grands romans en báihuà comme le Shuí Hǔ Zhùàn, le Jìn Píng Méi ou le $X i \vec{i}$ Yóu Jiprésentent les deux ordres, avec une nette tendance pour "V O Nég V" à 1 'emporter lorsque 1 'objet est un pronom personnel. I1 donne de nombreux exemples de verbes identiques qui acceptent les deux ordres, dans le Shǔ̌ Hŭ Quán Zhuàn. Puis il se livre à une comparaison entre le Hóng Lóu Mèng en 80 chapitres (zhน̌bén 脂本, ca. 1760) et ce1 ui en 120 chapitres (chéngyǐběn 程乙本, ca. 1792) pour constater que ce dernier - le plus répandu - présente seulement 1 'ordre pékinois "V Nég v 0", ce qui n'est pas le cas du zȟ̌bén, le plus ancien des deux. On y trouve en effet quelques constructions en " $v$ O Nég $v$ ", qui seront par la suite corrigées en "V Nég v O" dans 1 'édition de 1792. Exemples :

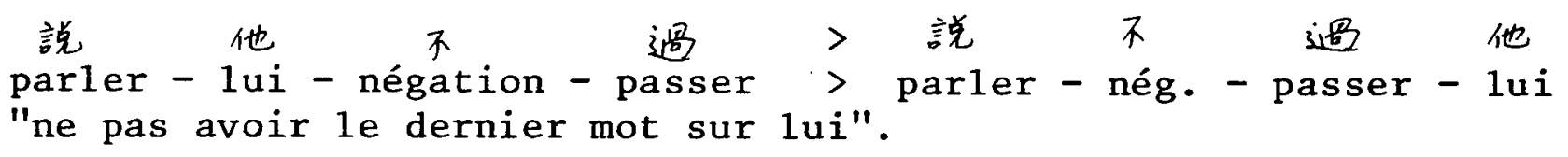

(16)

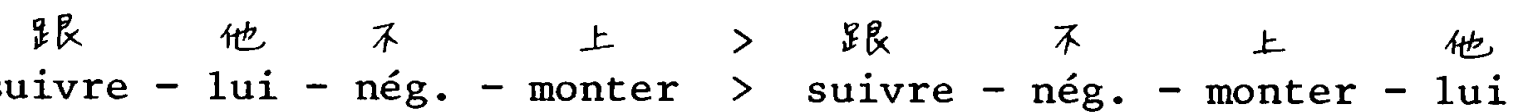
"ne pas arriver à le suivre (à suivre son rythme)".

（17）找他不是>找不着他 chercher - lui - nég. - voir > chercher - nég. - arriver à - 1ui "ne pas arriver à le trouver".

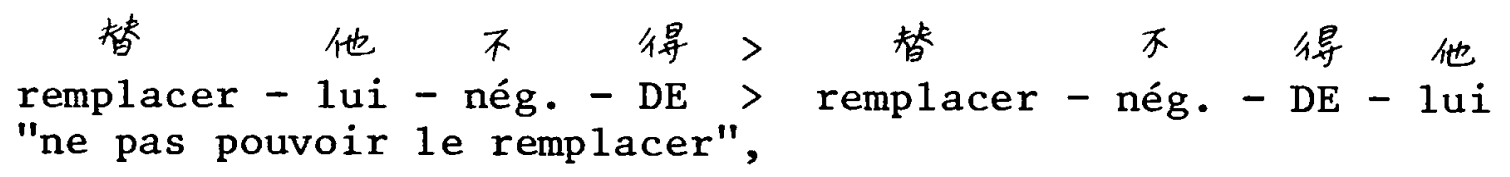

etc.

Miyata $\mathrm{I}$. conclut que cette unification en "V Nég $\mathrm{v} O$ " opérée dans le Hóng Lóu Mèng en 120 chapitres prouve que dans le pékinois de 1 'époque "V Nég $v 0$ " $s$ 'est déjà imposé et que "V $O$ Nég $v$ " y est ressenti soit comme archaĩque, soit comme marqué dialectalement. Il n'affirme pas nettement que cette unification s'est produite à Pékin entre le début des Qing et la deuxième moitié du XVIIle siècle. A vrai dire, il ne se prononce pas sur la question de savoir quand cette unification s'est produite; et il ne dit pas non plus si cette unification était ou non déjà effectuée dans le nord au moment où parurent les grands romans qui attestent une coexistence des deux ordres : le Shuz Hü Zhuàn, le Jin Ping Méi, etc.

Zhou Chiming (1958, p. 119) remarque simplement que 1 'ordre "V 0 Nég v" a dominé pendant les 600 à 700 ans qui vont des Song aux Ming et que ce n'est que sous les Qing qu'un changement progressif apparaît, qui imposera finalement 1 'ordre "V Nég v O".

3.2. Les documents du IVe siècle au XIIe siècle.

\section{a) 1es biànwén}

Ce sont des textes divers, souvent bouddhiques, découverts à Dunhuang, et donc antérieurs à 1015 (IXe - Xe siècles environ). 
Gen Sachiko (1985, p. 52) note que lorsqu'il y a un objet dans les tournures négatives "V $B U D E$ ", celui-ci vient toujours avant bridé 不得, et que les phrases de ce type présentent souvent une ambiguité, car il semble que l'interprétation où bude garderait son sens plein de "ne pas obtenir" est également possible. Voivi 1 'un des exemples qu'elle cite :

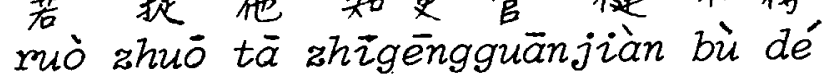

si - attrapper - lui - responsable aux horloges - nég. - DE

"Si on $n$ 'arrive pas à s'emparer du responsable aux horloges ...",

qui peut aussi être interprété comme "V 0 er 而 Nég DE (0)".

Zhou Chiming (1958, p. 218) ne cite, parmi ses exemples tirés des biànwén, que des constructions en "V O Nég v" (cf. plus haut 1'ex. (6)).

b) 1e zư Táng $\jmath^{\prime}$

Le Zü Táng Jí (Recueil de la salle des patriarches) date de 952. Cet ouvrage consiste en des biographies de moines bouddhistes et des dialogues qu'ils ont eus avec leurs disciples.

Notre décompte $n$ 'est pas exhaustif, mais pour 11 constructions en "V $O B U D E$ ", nous $\mathrm{n}$ 'en avons relevé aucune en "V $B U D E$ 0". Exemples :

（20）追市和尚㤢某甲不得（III，61）

shì yún héshàng guià móujiă bù dé

mâ̂tre - dire - moine - en vouloir - moi - négation - DE

"Le mâ̂tre dit : 'tu ne peux pas m'en vouloir".

（21）到与摩，時整。理脚手不得（IV，64）

dào yưmó shí zhénglì jǐ́o shóu bù dé

arriver - tel - moment - arranger - pieds - mains - nég. - DE

"En un tel moment, on ne sait plus où donner de la tête".

Pour ce qui est des constructions en "V $B U \mathrm{v}$ ", elles sont très nombreuses, mais pratiquement jamais accompagnées de complément d'objet post-verbal. Nous $n^{\prime}$ en avons guère qu'un exemple sûr de chaque ordre. Citons celui de "V Nég v O", encore nouveau à 1 'époque, et relevé aussi par Ōta Tatsuo (1958, p. 234) :

蝦 跳不出幖 (IV, 7 et 27)

$x i \bar{a}$ tì̄o bu chī dǒu

crevette - sauter - négation - sortir - récipient

"la crevette n'arrive pas à sauter hors du récipient".

c) le Dà Táng Sänzàng qư jīng shì hùa

Le Dà Táng Sānzàng qư jìng shì huà (Histoire agrémentée de poèmes de Sanzang des Tang parti en quête des soutras) (Xe siècle ?) est un texte écrit malgré tout en chinois assez classique. Il ne présente que des exemples de "V $O B U D E$ ", à 1 'exclusion de tout autre ordre. Exemple : 
(23) 開口吐之不得（IV, P. 13）

käai kouu tú zhì bù dé

ouvrir - bouche - recracher - le - négation - DE

"(E1le) ouvrit la bouche mais ne réussit pas à le recracher".

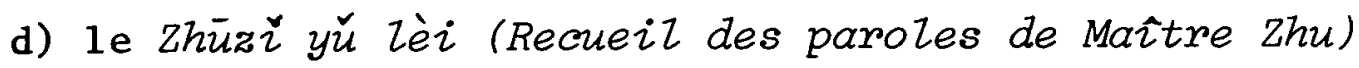

Nous avons utilisé une édition partielle, réalisée à partir du chénghuà běn 成化本 datant des Ming. Le texte, qui remonte à la fin du XIIe siècle, consigne les enseignements de Zhu Xi recueillis par ses disciples. Nous y avons relevé 11 "V 0 Nég $v$ " et une seule forme " $V$ Nég v O" (où le substantif qui suit $v$ est vraiment un objet). Dans les structures " $\mathrm{V} O \mathrm{Nég} \mathrm{v}$ ", sept d'entre elles ont un 0 qui n'est pas un pronom. Ex :

（24）不曾是他病虎説他不倒 (chap. 124, 55, p. 372) bù céng jiàn tà bingchù shuō tā bù dăo négation - voir - son - défaut - dire - lui - nég. - s'écrouler "C'est parce que vous n'avez pas vu ses défauts que vous pouvez avoir raison de 1 ui".

（25）都䘽 貼那原，道不起 （chap. 137，21，p.438）

dōu chèn tié ná yứn dào bù qž

tout - coller sur - ce - originel - voie - nég. - lever

"Et tout cela ne colle pas avec sa 'voie originelle"".

Quant à "V 0 Nég $D E$ ", nous en avons dénombré 13 exemples contre seulement un vrai "V Nég $D E$ O" ; mais il faut signaler ici la variante très fréquente en "Nég V $D E$ O" : 25 occurrences. Cette dernière forme n'est nullement limitée, dans ce texte, aux verbes de perception ou au verbes psychologiques (du type jide 記得"se souvenir"), ni aux cas où 1a négation est wéi 未 ou bucéng 不曾. Mais laissons de côté, pour le moment, cette dernière variante et le problème qu'elle pose, celui-ci étant trop vaste pour être traité en quelques 1 ignes. Exemples de "V 0 Nég $D E^{\prime \prime}$ :

添一字不得, 減一字不得（chap.19,59, p. 137)

tián yí zi bù dé, jiăn yí zì bù dé

ajouter - un - caractère - nég. - DE - enlever - un - caractère nég. - DE

"On ne peut ajouter ni ôter un seul mot".

$$
\text { 州乎字剖太字不得早 (chap.94,20, p. 219) }
$$

jiāng you zì xùn tài zì bù dé

prendre - 'you' - caractère - gloser - 'tai' - caractère - nég. -

DE

"On ne doit pas interpréter le mot 'you' comme étant le mot. 'tai'".

Les documents a) à d) relèvent d'un stade de développement du chinois que A. Peyraube (1984) appelle le "bas-médiéval" (pour lui, du VIe siècle à 1250) et qui se comporte, pour le point qui nous intéresse ici, de manière assez homogène, puisque les ordres "V 0 Nég v" ou 
"V O Nég $D E "$ (formes disjointes) sont, de loin, les plus courantes, et que 1 'ordre du pékinois actuel (formes groupées) n'apparât encore qu'à 1 'état embryonnaire. Le problème de la localisation des parlers chinois que reflètent ces documents n'est pas ici sous-estimé, mais nous ne disposons pas pour le moment d'éléments suffisants pour nous risquer à des affirmations simplificatrices.

3.3. Les documents des XIIIe et XIVe siècles.

Les documents qui suivent, e), f) et $g$ ), sont marqués dialectalement : e) est du pékinois, f) représente les dialectes du nord du Fleune Jaune, g) est une traduction du mongol. Comme nous allons le voir, ces textes ont une caractéristique commune : ils ne contiennent que des tournures groupées, c'est-à-dire qu'ils manifestent $1^{\prime}$ ordre du pékinois actuel "V Nég v O" ou "V Nég $D E$ O", et cela, quatre siècles environ, déjà, avant le Hóng Lóu Mèng.

e) le théâtre des Yuan. Devant la difficulté de trouver des pièces dans des éditions des Yuan qui comprennent suffisamment de passages parlés pour fournir des exemples, et dont 1 'auteur est connu et originaire de Dadu $大$ 都 ou tout au moins du nord, nous ne citons ici qu'une seule pièce de Guan Hanqing 雕嫨唧 (XIIIe siècle) : Bài Yué Tíng 段月亭 (Le pavizion avec vue sur la Zune). On y relève, dans les passages parlés, un exemple de "V Nég $D E O^{\prime \prime}$ et deux exemples de "V Nég $v$ O" :

$$
\begin{aligned}
& \text { （28）我以此上忘不下他 (p. 48) } \\
& \text { wŏ yǐ cř shiang wàng bu xì̀ tā } \\
& \text { je - pour - cela - sur - oublier - nég. - descendre - lui } \\
& \text { "C'est pour cela que je n'arrive pas à l'oublier". }
\end{aligned}
$$

Il s'agit bien, dans tous les cas, de l'ordre du pékinois actuel.

f) Lăo qĩdà yànjiěe et Piáo tōngshi yànjiě (Edition annotée de Le vieux sinologue) et (Edition annotée de Piao 1'interprète). Il s'agit de manuels de chinois parlé, à 1 'usage de marchands coréens se rendant en Chine du nord, qui reflètent sans doute la langue du XIVe siècle, bien que les éditions dont nous disposons à ce jour soient plus tardives (cf. Ōta Tatsuo, 1953).

Le caractère extrêmement parlé de ces textes ainsi que leur volume donnent une valeur certaine au fait que seul 1 'ordre du pékinois actuel y est attesté : 12 exemples de "V Nég $D E$ O" et 4 exemples de "V Nég v O". Nous n'avons trouvé aucune occurrence de formes disjointes. Exemples :

(29) 這的是恠不的人也㤢不的虫子(Piáo, 下, p. 262) zh̀̀de shi guiai bu de rén yé guài bu de chóngzi ceci - être - blâmer - nég. - DE - gens - aussi - blâmer - nég. $\mathrm{DE}$ - insecte

"De cela, on ne peut blâmer ni les gens ni les insectes". 
（30）官人。捨不的錢那裹買的（Piao,下, p. 312) guànrén shě bu de qián năli măi de monsieur - laisser - nég. - DE - argent - où - acheter - DE "Si vous êtes trop pingre, comment est-ce que vous pourriez 1 'acheter ?"

（31）依不得我時我不瞢（LaO, 下, p. 237） yi bu de wó shi wŏ bú mái dépendre - nég. - DE - moi - si - je - nég. - vendre "Si cela ne me convient pas, je ne vends pas".

（32）如今壹時出不上偠钱 (PiaO,上, p. 96) mijin mài shí chü bu shàng jiàqián maintenant - vendre - si - sortir - nég. - monter - prix "Si tu les vends maintenant, tu n'en tireras pas un bon prix".

（33）怎麼做不出一套衣裳来（Piao,中, p. 244) zènmo zuò bu chù yí tào yĩshang lai comment - faire - nég. - sortir - un - ensemble - vêtement - venir "Comment se fait-il que vous ne puissiez me faire un costume ?"

\section{g) Yuán Cháo Mìshì (L'histoire secrète des Mongols).}

Il s'agit bien sûr, ici, de la traduction chinoise globale et non du mot à mot de ce texte mongol décrivant la vie de Gengis Khan. C'est un chinois très mongolisé du point de vue grammatical (cf. Yamakawa Hidehiko, 1976), mais il reflète bien la langue parlée, sans doute du XIVe siècle environ. Exemples :

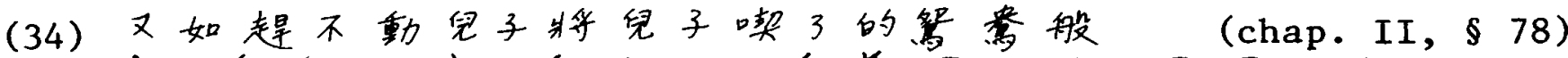
yòu rú găn bu dòng érzi jiāng érzǐ chì le de yuānyāng bān et - comme - chasser - nég. - bouger - fils - prép. - fils manger - part. aspect. - part. struct. - canard mandarin - pareil "Tel le canard mandarin qui, ne pouvant faire avancer ses fils, mange ses fils".

（35）… 喫不 3 這一简乳 (chap.XII, 244)

... chi bu liăo zhè yi ge mù manger - nég. - accomplir - ce - un - spécificatif - lait "(IIs) n'ont pas pu boire de ce lait".

Nous avons aussi trois occurrences de "V Nég $D E$ O". Exemple :

$$
\begin{aligned}
& \text { 我做 不得你右手 (chap. VIII, § 190) } \\
& \text { wó zù bu de nì yò shóu - } \\
& \text { je - faire - nég. - DE - toi - droit - main } \\
& \text { "Je ne peux pas être ton bras droit". }
\end{aligned}
$$

L'exemple qui suit semble être la seule forme disjointe de notre corpus ; il pourrait constituer un contre-exemple à ce que nous avons affirmé. Mais cet exemple est douteux. Pour notre part, nous 1 'interprétons comme deux propositions juxtaposées, notamment parce que le complément de lieu est avant le verbe : 


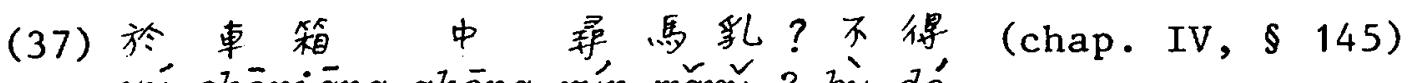

yú chēxiāng zhōng xún mă̌̌̀ ? bù dé

à - charrette - intérieur - chercher - lait de jument - ? - nég. obtenir (DE)

"Il chercha du lait de jument dans une charrette, mais sans succès" (7).

Si notre interprétation de ce dernier exemple est correcte, nous voilà donc avec un document de plus où seules les formes groupées, les mêmes qu'en pékinois actuel, apparaissent, à l'exclusion des formes disjointes, et cela dès la fin du XIVe siècle.

\section{CONCLUSION}

L'unification en un ordre groupé des constructions "V Nég v O", "V Nég $D E$ O", déjà constatée par Miyata I. dans le Hóng Lóu Mèng (fin du XVIIIe siècle) était en fait déjà achevée en chinois septentrional, près de quatre siècles plus tôt, vers la fin du XIVe siècle (fin des Yuan, début des Ming).

Le fait que de nombreux romans en báihuà présentent les deux ordres "V O Nég V" et " $V$ Nég $v$ O" en coexistence ne tient donc pas à 1 'ancienneté de la date à laquelle ils ont été rédigés, mais à la langue - dialectalement marquée - que leurs auteurs ont utilisée. Les dialectes du Bas-Yangzi ont en effet conservé cette forme disjointe "V O Nég V". Et il va sans dire que le rôle immense qu'a joué le "mandarin du sud" dans les oeuvres littéraires pendant plusieurs siècles n'est sans doute pas indifférent à cet état de choses (8).

Le changement d'ordre "V O Nég $D E$ (ou v)" > "V Nég $D E$ (ou v) O" doit être replacé dans le cadre d'une vaste réorganisation des compléments postverbaux (les büyú 補語 du chinois contemporain, mais aussi les objets dont ils n'étaient pas si évidemment distingables), qui vise, dans les cas qui nous préoccupent ici, à différencier l'expression syntaxique du simple "non réalisé" de celle de l'"irréalisable". Cette différenciation dépendait avant les Song plutôt du contexte et des relations sémantiques entre les constituants, 1 a construction elle-même restant ambiguë.

Un phénomène semblable a eu lieu pour les formes affirmatives de ces constructions, aboutissant à la différentiation actuelle en complément

(7) Dans ces exemples du Yuán Cháo Mishř, nous avons emprunté à P. Pe1liot (1949) la traduction de l'exemple (34).

(8) Les différences qu'a notées Miyata I. entre les éditions du Hong Lou Meng devraient ainsi plutôt être comprises en termes de différences entre les idiolectes de Cao Xueqin, auteur présumé des 80 premiers chapitres, et de Gao E, auteur présumé des 40 derniers, la famille de Cao étant installée depuis longtemps dans le sud alors que Gao était Pékinois. La différence de générations a sans doute beaucoup moins joué. Mais trop de paramètres entrent en jeu pour que nous nous risquions à une telle simplification. 
de possibilité (kěnéng bǔyŭ 可能補語) du type chr̃debăo 吃得飽"pouvoir manger à sa faim" / chïbubăo 吃不飽 "ne pas pouvoir manger à sa faim" et en complément résultatif (jiéguó bǔyŭ 結果補語) du type chi de hěn băo 吃得很飽 "être rassasié" / chi de bu tài băo 吃得不太館 "n'être pas rassasié", avec des contraintes sur les formes en "V $D E$ $O v^{\prime \prime}$ où $O$ est pivot, qui obligent maintenant à recourir au redoublement du verbe ou à 1 'antéposition en $B A$ 把 (9).

Mais l'évolution de ces formes affirmatives requiert à elle seule une étude séparée, et nous finirons par une remarque de Lü Shuxiang (1944, pp. 66-67) sur les formes en "V $D E O \mathrm{v}$ ", dont voici un exemple :

$$
\begin{aligned}
& \text { 若説得這顽親事成（京本通俗小説 13） } \\
& \text { miò shuō de zhè tóu qinshi chéng } \\
& \text { si - parler - DE - cette - spécif - union - se réaliser } \\
& \text { "Si on tombe d'accord pour que cette union se réalise ..." }
\end{aligned}
$$

Lû Shuxiang nous dit que de telles formes étaient courantes, en tant que formes affirmatives, du temps des Tang et des Song; or, elle n'expriment maintenant que 1e "réalisé" (yž chéng 已成), c'est-à-dire le résultat ; pour exprimer le "non encore réalisé" (wèi chéng 未成), c'est-à-dire le potentiel (kĕnéng可能), on a recours à 1 'ordre "V $D E$ v O". Lü Shuxiang donne les exemples de chidexià fàn 喫得下领 "arriver à manger" qui ne peut plus se dire chì de fàn xià 喫得领下, ainsi que ceux de 1'opposition entre (39) et (40):

$$
\begin{aligned}
& \text { 喫得一鈵空 } \\
& \text { chi de yi guö köng } \\
& \text { manger - DE - un - marmite - vide }
\end{aligned}
$$

(40) 喫得空 - 鍶

chĩ de kōng y $\vec{\imath}$ guó

manger - $\mathrm{DE}$ - vide - un - marmite

où (39) exprime le réalisé : "achever la marmite" et (40) le réalisable : "pouvoir finir la marmite". Mais (40) a la même structure que (39) qui, à l'époque, n'avait pas ce sens de "réalisé" par opposition au "réalisable".

La forme affirmative comme la forme négative de telles constructions a été au coeur de la restructuration du système syntaxique qu'a connu le chinois au cours de ces quelques siècles, qui a permis de lever $1^{\prime}$ ambiguité pesant sur la marque de 1 'opposition "réalisé / réalisable", en donnant à ces deux catégories une expression syntaxique distincte.

(9) Sur le statut de tels compléments de résultat où 0 est pivot, cf. Sòng Yùzhù (1981) qui a consacré un article sur les constructions à pivot en $D E$. 
C'est cette même ambiguïté "réalisable" / "réalisé" que montre 1'emploi de $D E$ en langue vernaculaire ancienne.

Christine LAMARRE

C.R.L.A.O.

\section{SUMMARY}

This paper aims to show when and where occured the syntactic change which led from the word order "Verb + Object + negation + Resultative postverb" (here "V O Nég $v "$, ex : shuō tā bú guò), prevailing in Tang times, to the present Pekingese word order "Verb + negation + Resultative postverb + object" (here "V Neg $v 0$, ex : shuō bu guò tā).

While both orders are met in 'baihua' Ziterature of Ming times, we argue - through an investigation of Tang, Song, and Yuan texts - that the present Pekingese word order was the only attested one in the Northern dialect as soon as the Yuan period; the ancient word order "V $O$ Neg $v^{\prime \prime}$, however, is still very common in many southern dialects, which probably accounts for the coexistence of "V $O$ Neg $v$ " and "V Neg $v$ " in Ming texts.

This word order change is linked to the one that occuring later in corresponding affirmative constmuctions. The significance of both changes lies in the fact that Modern Pekingese is thus provided with two distinct stmuctures unambiguous as for resultative and potential meaning, while the previous constructions were basically resultative acquiring only in some limited contexts a potential meaning.

\section{Addendum}

Dans un article tout récent consacré à de 得 dans le dialecte de Changsha, Zhāng Dàí 張大旗 donne de nombreux exemples de 1 'ordre "V Nég O v", cité par ailleurs par Yuan Jiahua (1983, p. 321) pour le Gan et par Zhăn Bóhui (1981, p. 78) pour le dialecte de Suzhou.

Cf. Zhāng Dàqí (1985), "Chángshähuà 'de'zì yánjiū" 長沙話“得”字研究 Fängyán 1, pp. 46-63. 


\section{CORPUS}

Dünhuáng biànwén ji 敦煌㘘文集 (Recueir de 'bianwen' de Dunhuang). Pékin : Renmin wenxue chubanshe, 1957, 2 tomes, 922 p.

Zú táng jí 祖堂集 (Recueil de la salle des patriarches). Fac-similé d'une édition de 1245, réédition de 1974 (1ère édition : 1971). Kyoto : Chübun Shuppansha, $385 \mathrm{p}$.

Dà Táng Sānzàng qù jîng shí huà 大唐三藏取絰詩話 (Histoire agrémentée de poèmes de Sanzang des Tang parti en quête des soutras). Shanghai : Gudian wenxue chubanshe, 1954, 46 p.

Zhüzĭ yü lèi 朱子語類 (Recueil des paroles de Mấtre Zhu). Edition traduite et annotée, partielle, réalisée par une équipe de 1 'Université de Kyushu, à partir de 1 'édition 成化本 des Ming. Tokyō : Meitoku Shuppansha, 1981, 516 p.

Xĩn jiào Yuán kăn záju sănshi zhŏng 新校元利雜劇三十種 (Trente pièces de théâtre des Yuan, nouvellement révisées). Pékin : Zhonghua shuju, 1980,2 tomes, $807 \mathrm{p}$.

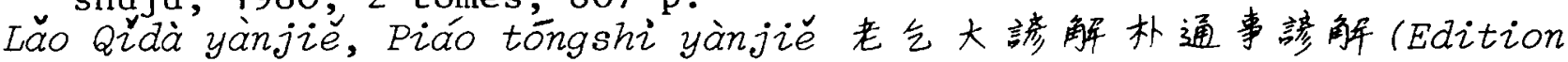
annotée du 'Vieux sinologue'. Edition annotée de 'Piao l'interprète'). Taibei : Lianjing chubanshe, 1978, $408 \mathrm{p}$.

Yuán cháo mishi 元朝秘史 (Histoire secrète des Mongols). Texte en XV chapitres. Moscou : Académie des Sciences d'URSS (Izdatel'stvo vostochnoi 1iteratury), 1962,602 p.

\section{REFERENCES BIBLIOGRAPHIQUES}

FU Guotong (1961), "Wuyihua li de yixie yuyin yufa xianxiang 武義話裹 的一些語音語法现象 (Quelques phénomènes phonétiques et grammaticaux $\mathrm{du}$ parler de Wuyi)" Zhongguo yuwen (Beijing) $\mathrm{n}^{\circ}$ 9, pp. 30-31.

GEN Sachiko 玄幸子 (1985), Tonko henbun ni okeru "V DE" ni tsuite 敦煌 变文に於ける“ $v$ 得”に二ne (A propos de "Verbe + DE" dans les 'bianwen' de Dunhuang). Mémoire de maîtrise (non publié) de l'Université de jeunes filles de Nara, 145 p.

HASHIMOTO Mantaro 橸本萬太郎 (1978), Gengo ruikei chiri ron 言語類型地 理言侖 (Géographie typologique des langues). Tokyo : Kobundo, $257 \mathrm{p}$.

LU Shuxiang 吕叔湘 (1944), "Yu dongci hou DE yu BU youguan zhi cixu wenti 與動詞後得與不有墽之詞序閣題 (Problèmes d'ordre des mots en rapport avec DE et BU en position postverbale)" in Hanyu yufa Zunwen ji 漠語 語法論文集 (Recueil d'articles en grammaire chinoise). Pékin : Wenzi gaige, 1955, pp. 59-68.

MIYATA Ichiro 宫田一郎 (1973), "KoZomu no kotoba ni tsuite「兹棲萝,のニとげ != $\cdots$ e (A propos de la langue du Hong lou meng)" Jinbun kenkyu (Osaka) $\mathrm{n}^{\circ} 25-3$, pp. 39-53.

OTA Tatsuo 太田辰夫(1953), "Rokitsudai no gengo ni tsuite 老乞大の言語 : $n$ v 2 (A propos de la langue de Lao Qida)", in Chugokugogaku Kenkyukai Ronshu中国語学研究會論集 (Nara), pp. 1-14.

--- (1958), Chugokugo rekishi bunpo 中国語嚰史丈法 (Grammaire jistorique du chinois). Tokyo : Konan Shoin, $439 \mathrm{p}$.

PELLIOT Paul (1949), Histoire secrète des Mongols. Traduction des chapitres I à VI. Paris : A. Maisonneuve, 196 p. 
PEYRAUBE Alain (1984), Syntaxe diachronique du chinois : évolution des constructions datives du XIVème siècle avant J.C. au XVIIIème siècle. Thèse de Doctorat d'Etat. Université de Paris VII.

SONG Yuzhu农玉柱 (1981), "Lun dai DE jianyushi論带得兼語式 (A propos des constructions à pivot en DE)", in Xiandai hanyu yufa Zunji 現代 漢語語法論集(Recueil d'articles sur la grammaire du chinois contemporain). Tianjin : Tianjin renmin chubanshe, pp. 68-75.

YAMAKAWA Hidehiko 山川英产 (1976), "Genko hishi soyaku goho sakki 元朝 秘史総訳語法札記 (Notes grammaticales sur la traduction globale de 1'Histoire secrète des Mongols)", Nagoya Daigaku Bungakubu kenkyu Ronshu (bungaku) (Nagoya) $n^{\circ}$ LXVII, pp. 63-79.

YUAN Jiahua 袁家驧 (1960, éd? Révisée 1983), Hanyu fangyan gaiyao 漠語 方言概要 (Aperøu des dialectes chinois). Pékin : Wenzi gaige, $323 \mathrm{p}$.

ZHAN Bohui 冬伯慧 (1981), Xiandai hanyu fangyan 现代漠語方言 (Les dialectes chinois contemporains). Wuhan : Hubei renmin chubanshe, $211 \mathrm{p}$. ZHOU Chiming 周䐅明 (1958), "Hanyu de shidongxing fushi dongci 漢語的 使動性複式動劾 (Les verbes complexes causatifs du chinois)", in Hanyu luncong (Wenshizhi vol. 4). Pékin : Zhonghua shuju, pp. 175-226. 